\title{
Algorithms for Service Differentiation in MAC Layer over MANETs
}

\author{
Kwan-Woong Kim ${ }^{1}$, Sung-Hwan $\mathrm{Bae}^{2}$, and Dae-Ik Kim ${ }^{3}$ \\ ${ }^{1}$ Division of Electrical Electronic \& Information Engr., Wonkwang Univ., Iksan, 570-749, \\ Republic of Korea \\ watchbear@wonkwang.ac.kr \\ ${ }^{2}$ Dept. of Multimedia, Information \& Telecommunication Engr., Hanlyo Univ. Gwangyang, \\ 545-704, Republic of Korea \\ hlu008@hanmail.net \\ ${ }^{3}$ Dept. of Semiconductor Materials \& Devices Engr., Chonnam Nat'1 Univ., Yeosu, 550-749, \\ Republic of Korea \\ daeik@chonnam.ac.kr
}

\begin{abstract}
Currently, the IETF group is working on service differentiation in the Internet. However, in wireless environments such as ad hoc networks, where channel conditions are variable and bandwidth is scarce, the Internet differentiated services are suboptimal without lower layers' support. The IEEE 802.11 standard for Wireless LANs is the most widely used WLAN standard today. It has a mode of operation that can be used to provide service differentiation, but it has been shown to perform badly. In this paper, a new scheme of service differentiation to support QoS in the wireless IEEE 802.11 is proposed. It is based on a multiple queuing system to provide priority of user's flow. Compared with the original IEEE $802.11 \mathrm{~b}$ protocol, the proposed scheme increases overall throughput in the MAC layer.
\end{abstract}

\section{Introduction}

A mobile ad hoc network (MANET) [1] is an autonomous distributed system that consists of a set of identical mobile nodes that move independently and freely. Each node communicates over relatively bandwidth-constrained wireless links with other nodes that reside within its transmission range. Because of limited radio propagation range, mostly routes are multi-hop.

Ad hoc networks are useful in many applications because they do not need any infrastructure support. Ubiquitous sensor networks, disaster recovery, rescue and automated battlefields are examples of application environments. Without no base station or centralized administration, the nodes are free to move randomly and organize themselves arbitrarily. Thus, the networks topology may change rapidly and unpredictably.

In general, the network needs are governed by the service requirements of end user applications. The network is expected to guarantee a set of measurable pre-specified service attributes to the users in terms of end-to-end performance, such as bandwidth, delay, packet loss and jitter. Quality of Service (QoS) in this case is considered as a set of service requirements that needs to be met by the network while transporting a 
packet stream from a source to its destination. The ability to provide an adaptive QoS in mobile environment is a key to the success of next generation wireless comm.unications systems.

Currently, the IETF group is working on service differentiation in the Internet. However, in wireless environments such as ad hoc networks, where channel conditions are variable and bandwidth is scarce, the Internet differentiated services are suboptimal without lower layers' support. The IEEE 802.11 standard for wireless LANs has a mode of operation that can be used to provide service differentiation, but it has been shown to perform badly.

Many works on service differentiation have been carried out, especially via distributed mechanisms, as it is the case in [2], [3] and [4]. All these works have focused on the problem of how to differentiate services by differentiating priority of the access to the wireless channel. In our approach, instead of access priority differentiation, a service differentiation mechanism is proposed to handle packets over a class queuing system in the MAC layer. Also a new packet discarding policy is presented for router function of a mobile node to enhance end-to-end performance.

In Sect. 2, a new service differentiation scheme based on a multiple queuing system is proposed. In Sect. 3, Simulation results obtained by the proposed scheme are evaluated. Finally, Sect. 4 presents some conclusions.

\section{Proposed Algorithm}

In pure IEEE $802.11 \mathrm{~b}$ MAC, single queue is used in best-effort manner and it has no capability to support QoS such as bandwidth guarantee, delay and loss rate. Packet Discarding Mechanism (PDM) can be used to manage the buffers in the mobile device. Under these mechanisms, the information carried by high priority packet is considered to be more important than the information carried by low priority packet. In order to achieve certain level of loss performance, PDM with the priority queue can be used to reduce the loss rate of high priority packets at the expense of higher loss rate of low priority packets.

In this paper, an adaptive weighted round robin (WRR) scheme is proposed to provide service differentiation in multiple class queuing system. We define two classes of traffic; low priority for best effort traffic and high priority for multimedia traffic to simplify the mechanism. The main feature of the proposed algorithm is that it regulates the weight of high priority class according to traffic load. Regulation of the weight of classes makes it enable to guarantee minimum throughput of best-effort traffic under severe congestion. The proposed queuing system is shown in figure 1 .

When a packet arrives from the MAC layer, the packet is accepted to the associated class queue with its priority. To adjust the weight of classes to traffic loads, two thresholds and the length of the high priority class queue are used. Each weight of class queues is defined as

$$
\begin{aligned}
& W_{H}=W_{\text {initial }}, W_{L}=1-W_{H}, \text { when } Q[h]<T_{L} \\
& W_{H}=W_{\text {initial }}+k \cdot \min \left(\frac{Q[h]-T_{L}}{T_{H}-T_{L}}, 1\right), W_{L}=1-W_{H}, \text { when } T_{L} \leq Q[h]
\end{aligned}
$$




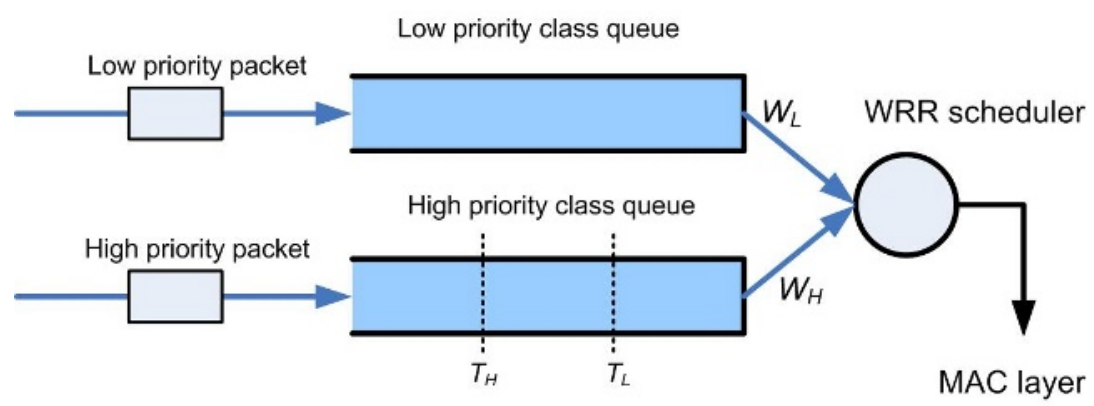

\section{$W_{H}$ : Weight of high priority flows \\ $W_{L}:$ Weight of low priority flows}

Fig. 1. Queuing model of the proposed algorithm

where $Q[h]$ is the length of the high priority class queue, $W_{H}$ is the weight of the high priority class queue and $W_{L}$ is the weight of the low priority class queue. $T_{L}$ is low threshold of the high priority class queue and $T_{H}$ is high threshold of the high priority class queue. $W_{\text {initial }}$ is the initial weight of high priority class queue and $k$ is the constant. The weight of the high priority class queue, $W_{H}$, is varied from $W_{\text {inital }}$ to $W_{\text {intial }}+k$. Weighted round robin (WRR) scheduler chooses the queue according to its weight and transmits packet from the queue to MAC.

\section{Packet Discarding Policy (PDP)}

In Ad hoc network, each mobile node acts as router as well as host. There are two kinds of packets into interface queue $\left(\boldsymbol{I} \boldsymbol{F}_{-} \boldsymbol{q}\right)$; packets from higher layer (transport layer) and packets from MAC layer. Packets from MAC layer traverse intermediate nodes to reach their destinations. Because they have consumed wireless channel resources, we consider that packets from MAC layer are more important than those from higher layer.

If the buffer overflow occurs in $I F_{-} \boldsymbol{q}$ caused by excessive traffic of higher layer, the IF_q will discard all incoming packets including packets from MAC layer. Discarding packets come from MAC layer results in waste of wireless channel resources. To overcome this inefficiency, a packet discarding policy (PDP) that protects packets come from MAC layer under buffer overflow is proposed. It tries to keep available buffer space for packets from MAC layer by discarding packets from higher layer. The goal of PDP is to protect packets that traverse multi-hops to reach their destination, to improve overall end-to-end performance and to increase wireless channel utilization.

When a new packet $p k t$ arrives at $I F_{-} \boldsymbol{q}$, behavior of the proposed mechanism is as shown in below. scr_addr is source address of packet pkt, addr_ is address of this node and $Q[l]$ is the length of the low priority class queue. 


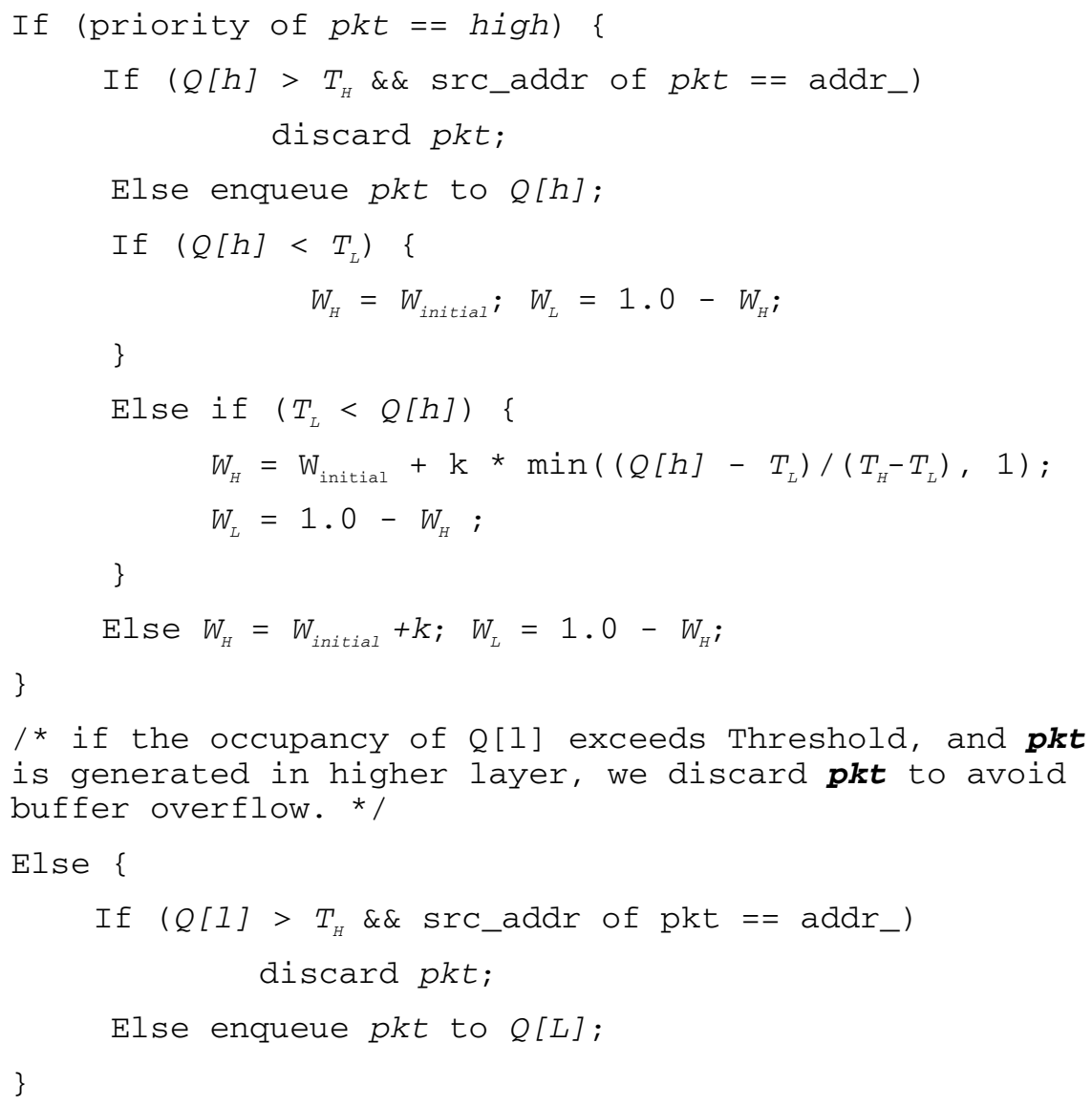

\section{Experimental Results}

NS2 simulator [5] is used to make performance comparison between the proposed scheme and the drop-tail queue scheme. NS2 is a freely available discrete-event object-oriented network simulator. It provides a framework for building a network model, specifying data input, analyzing data output and presenting results.

The ad hoc network model used for simulation is shown in figure 2. It consists of 10 mobile nodes and 8 constant bit rate (CBR) traffic sources. Each CBR source generates packet every interval time. Interval time between packets is varying from 0.05 to 0.1 second. The packet size is set to $1 \mathrm{~K}$ bytes. Half of traffic sources are high priority class and the others are low priority class. Ad hoc on demand distance vector routing protocol is used and IEEE 802.11 is used for wireless MAC protocol. To avoid the bias of random number generation, we execute simulation 10 times for each configuration. A simulation time is set to 500 seconds. In the network model, CBR flows 1, 3, 4 and 6 are set to have high priority and the other flows are set to have low priority. We assign 25 packets to each class queue size for the proposed queuing system and 50 packets to drop-tail queue system. 


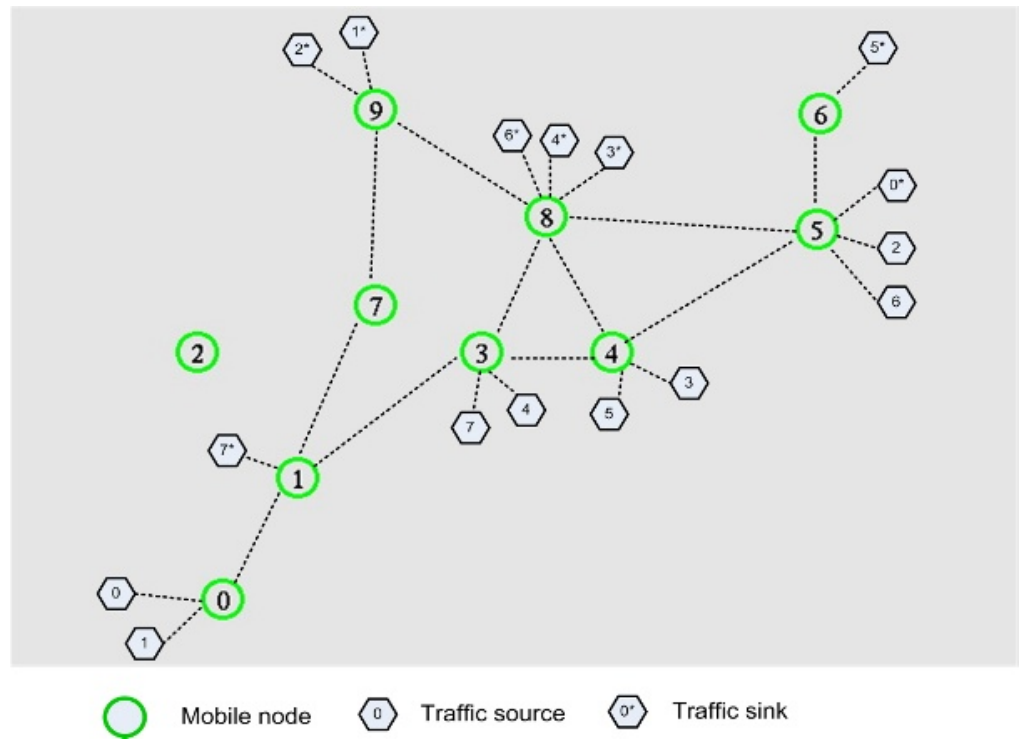

Fig. 2. Ad hoc network model for simulation

Figure 3 shows average number of received packets at CBR receivers. It can be seen that the proposed scheme can increase the end-to-end performance from 8\% $40 \%$ by compared to drop-tail queuing system. The reason is that the PDP of the proposed scheme increases end-to-end throughput by protecting packets that traverse multi-hop path from packet dropping caused by buffer overflow in intermediate nodes.

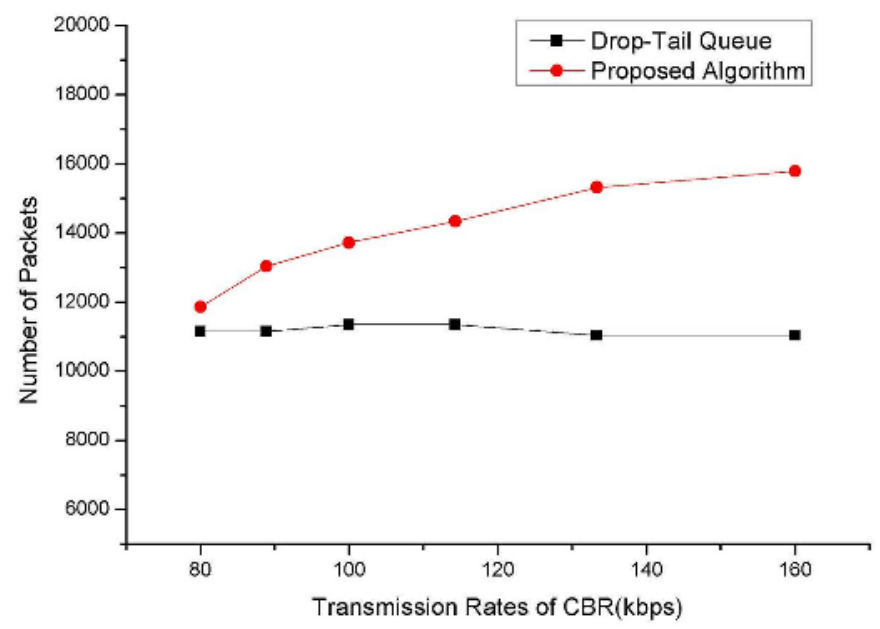

Fig. 3. Average number of received packets 
Figure 4 depicts average number of received high priority packets and average number of received low priority packets. It can be noticed that high priority packets are much more served than low priority packets. Moreover, the number of received high priority packets is increased as traffic load of high priority CBR is increased. Indeed the weight of class queues is dynamically varied with load of high priority flows. As the number of packets waiting in the high priority class queue is increased, high priority packets are more served. As shown in figure 4, results show that the drop-tail queuing system is incapable of being differentiate QoS.

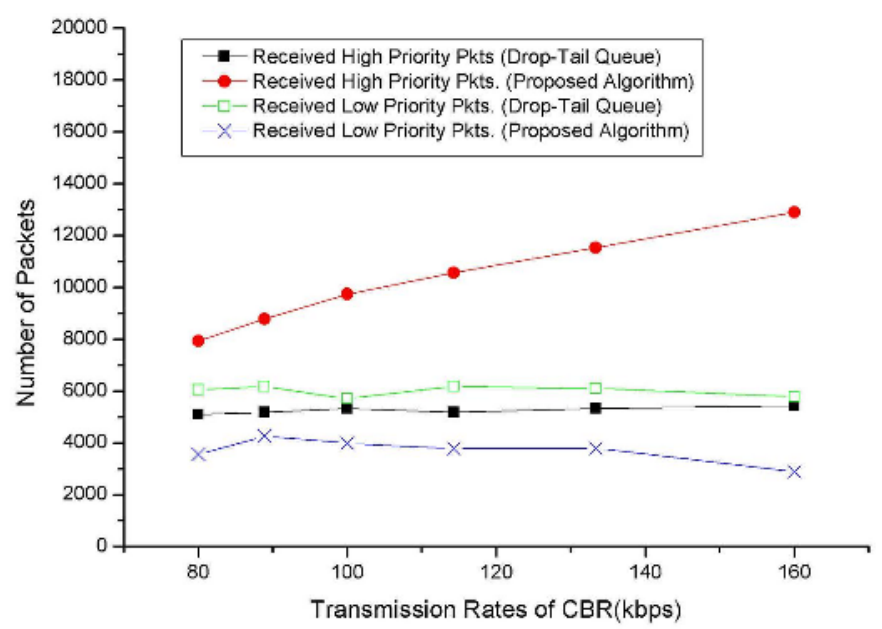

Fig. 4. Average number of received high priority \& low priority packets

Figure 5 plots average number of lost high priority packets and average number of lost low priority packets. The proposed scheme provides very low packets loss rate

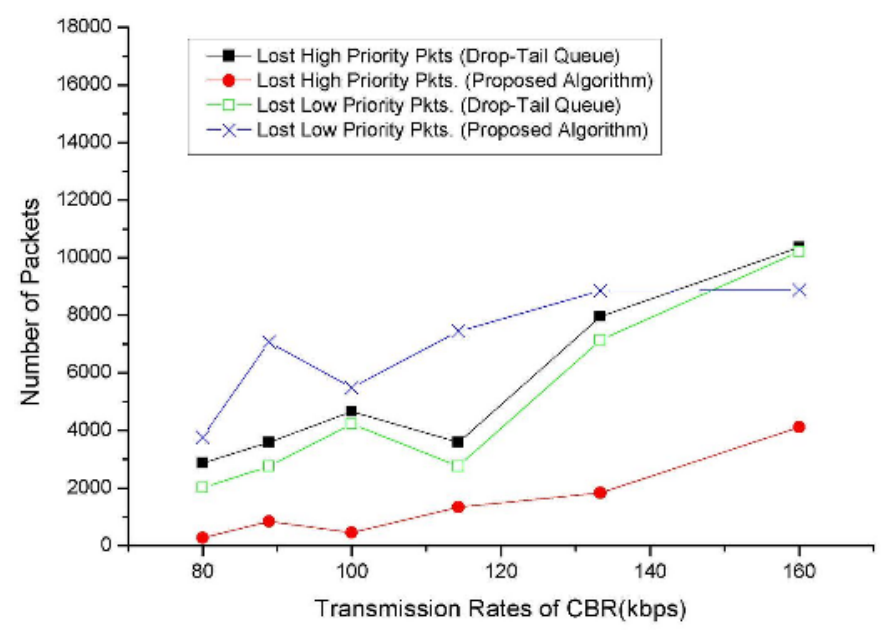

Fig. 5. Average number of lost high priority \& low priority packets 
for high priority packets compared to drop-tail queue scheme. The reason is that WRR scheduler tries to serve more high priority packets than low priority as high priority traffic increase.

Figure 6 shows end-to-end delay of high priority and low priority packets. Obviously, when traffic load is low, end-to-end delay of high priority packet keeps low. But traffic load become heavy, delay also becomes large. As shown in figure 6, WRR scheduler of the proposed scheme can provide better performance than drop-tail queue in terms of end-to-end transmission delay.

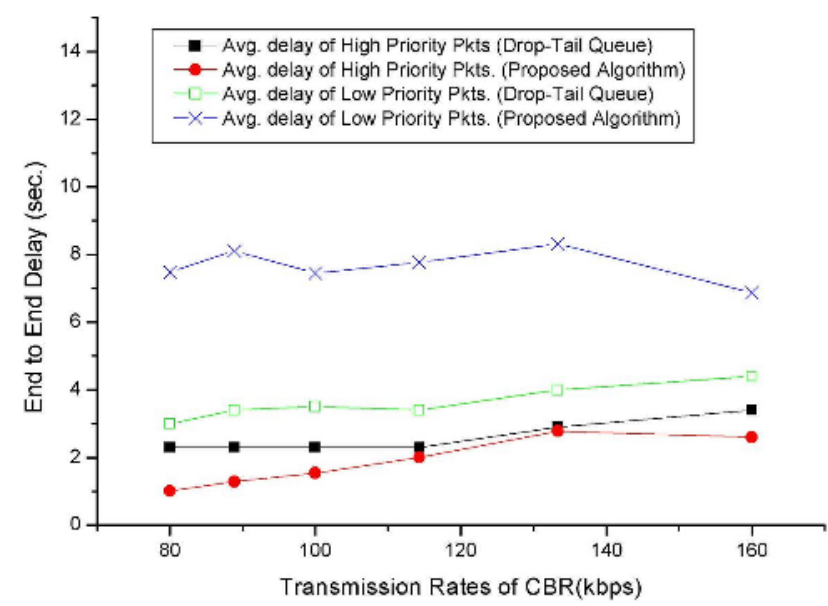

Fig. 6. End-to-end delay of high priority and low priority packets

Experimental results are quite positive in the sense that IEEE 802.11 MAC protocol with the proposed scheme outperforms than pure IEEE 802.11 MAC in all cases. Using our technique, the proposed scheme may improve overall end-to-end throughput as well as support service differentiation over multi-hop MANETs.

\section{Conclusions}

This paper has described a new service differentiation scheme based on a multiple queuing system and a PDP. The main feature of the proposed algorithm is that it regulates the weight of high priority class according to traffic load. A new PDP tries to keep available buffer space for packets from MAC layer by discarding packets from higher layer and protects packets that traverse multi-hops to reach their destination.

Simulation results show that the proposed scheme may improve overall end-to-end throughput as well as support service differentiation over multi-hop MANETs.

\section{References}

1. C. E. Perkins, Ad Hoc Networking. Addison-Wesley, Upper Saddle River, NJ, USA, Jan 2001.

2. I. Aad and C. Castelluccia, "Differentiation mechanisms for IEEE 802.11," Proc. of IEEE INFOCOM 2001, pp. 209-218. 
3. M. Barry, A. T. Campbell, and A. Veres, "Distributed Control Algorithms for Service Differentiation in Wireless Packet Networks," Proc. of IEEE INFOCOM 2001, pp. 582-590.

4. G. Bianchi and I. Tinnirello, "Analysis of Priority Mechanisms based on Differentiated Inter-Frame Spaces in CSMA/CA," in Proc. IEEE VTC 2003, vol. 3, pp. 1401 - 1405, Orlando (FL), Oct 2003.

5. NS-2 homepage: http://www.isi.edu/nsnam/ns. 